\title{
Association of school hours with outcomes of out-of- hospital cardiac arrest in schoolchildren
}

\author{
Akira Yamashita, ${ }^{1,2}$ Hisanori Kurosaki, ${ }^{1}$ Kohei Takada, ${ }^{1}$ Yoshio Tanaka, 1,3 \\ Yoshitaka Hamada, ${ }^{4}$ Tetsuya Ishita, ${ }^{5}$ Minoru Kubo, ${ }^{6}$ Hideo Inaba ${ }^{\circledR}$
}

\begin{abstract}
${ }^{1}$ Department of Circulatory Emergency and Resuscitation Science, Kanazawa University Graduate School of Medicine, Kanazawa, Japan ${ }^{2}$ Department of Cardiology, Noto General Hospital, Nanao, Japan

${ }^{3}$ Emergency Medical Centre, Ishikawa Prefecture Central Hospital, Kanazawa, Japan ${ }^{4}$ Department of Neurosurgery, Suzu General Hospital, Suzu, Japan

Department of Surgery, Kaga Medical Centre, Kaga, Japan ${ }^{6}$ Ishikawa Prefectural Nursing School, Kanazawa, Japan
\end{abstract}

\section{Correspondence to} Professor Hideo Inaba, Department of Circulatory Emergency and Resuscitation Science, Kanazawa University Graduate School of Medicine, Kanazawa, Ishikawa 9208641, Japan; hidinaba@med. kanazawa-u.ac.jp

Part of this study was presented at ERC Congress 2018, 21 and 22 September, Bologna, and its abstract was published in Supplement Issue of Resuscitation as e-publication.

Received 23 July 2019 Accepted 26 July 2019

\section{ABSTRACT \\ Objective To investigate the association of school hours with outcomes of schoolchildren with out-of- hospital cardiac arrest (OHCA).}

Methods From the 2005-2014 nationwide databases, we extracted the data for 1660 schoolchildren (6-17 years) with bystander-witnessed OHCA. Univariate analyses followed by propensity-matching procedures and stepwise logistic regression analyses were applied. School hours were defined as 08:00 to 18:00.

Results The neurologically favourable 1-month survival rate during school hours was better than that during non-school hours only on school days: $18.4 \%$ and $10.5 \%$, respectively. During school hours on school days, patients with OHCA more frequently received bystander cardiopulmonary resuscitation (CPR) and public access defibrillation (PAD), and had a shockable initial rhythm and presumed cardiac aetiology. The neurologically favourable 1-month survival rate did not significantly differ between school hours on school days and all other times of day after propensity score matching: $16.4 \%$ vs $16.1 \%$ (unadjusted OR 1.02; $95 \% \mathrm{Cl} 0.69$ to 1.51 ). Stepwise logistic regression analysis during school hours on school days revealed that shockable initial rhythm (adjusted OR 2.44; 95\% Cl 1.12 to 5.42 ), PAD (adjusted OR 3.32; $95 \%$ Cl 1.23 to 9.10), non-exogenous causes (adjusted OR 5.88; 95\% Cl 1.85 to 20.0) and a shorter emergency medical service (EMS) response time (adjusted OR 1.15; 95\% Cl 1.02 to 1.32) and witnessto-first CPR interval (adjusted OR 1.08; $95 \% \mathrm{Cl} 1.01$ to 1.15) were major factors associated with an improved neurologically favourable 1-month survival rate.

Conclusions School hours are not an independent factor associated with improved outcomes of OHCA in schoolchildren. The time delays in CPR and EMS arrival were independently associated with poor outcomes during school hours on school days.

\section{INTRODUCTION}

Although the incidence of out-of-hospital cardiac arrest (OHCA) in school-age children is lower than that in adults, OHCA in schoolchildren requires early and proper basic life support (BLS), including detection, emergency call and cardiopulmonary resuscitation (CPR). ${ }^{1-5}$ Furthermore, sudden cardiac arrest of presumed cardiac aetiology is not rare among school-age children and such OHCA is frequently associated with the sudden onset of ventricular fibrillation/tachycardia requiring defibrillation. ${ }^{346}$ In addition to preventative strategies for OHCA due to exogenous causes, including trauma, submersion, poisoning and suicide attempts, early initiation of CPR after recognition of cardiac arrest by bystanders and early defibrillation are important determinants of survival after OHCA in school-age children. ${ }^{78}$

Schools are responsible for the management of medical emergencies, including OHCA, in schoolchildren. OHCA in schoolchildren at school during school hours is mainly witnessed by school staff and students. ${ }^{9}$ In Japan, the number of automated external defibrillators (AED) installed at schools has continued to increase since their use by citizens was legally approved in July 2004. AEDs were installed at school in combination with the provision of CPR training to school staff members. ${ }^{70-13}$ Furthermore, BLS is widely taught to schoolchildren in Japan.

This study aimed to investigate whether school hours or school days are associated with improved outcomes of OHCA in schoolchildren. Furthermore, this study clarified major factors associated with neurologically favourable survival after bystander-witnessed OHCA in schoolchildren.

\section{METHODS}

\section{Study design and setting}

Consent was obtained from the Japanese Fire and Disaster Management Agency (FDMA) to analyse nationwide OHCA data in the All-Japan Utstein Registry of FDMA collected from 1 January 2005 to 31 December 2014.

Statistical data of Japanese schools are published by the Japanese Ministry of Education, Culture, Sports, Science and Technology. The Japanese school system comprises 6 years of elementary school, 3 years of junior high school and 3 years of high school. Elementary and junior high schools are compulsory education, and the rate of continuous education during the study period exceeds $97 \%$. In 2014, the population of Japan was 127 million with a school-age children population of 13.5 million (6-17 years), constituting $10.7 \%$ of the total population. The number of elementary, junior high and high schools was 20 852, 10557 and 4963, respectively.

The Japanese emergency medical service (EMS) responds to all requests for ambulance dispatch. The FDMA has provided standard guidelines for dispatcher-assisted telephone CPR instructions. ${ }^{14}$ Unless a patient with OHCA is obviously dead or presents postmortem changes, EMS personnel must continue resuscitation at the scene. In addition to BLS with AED application, paramedics in ambulance teams are allowed to perform several advanced life support procedures, including suprapharyngeal airway and peripheral venous infusion 


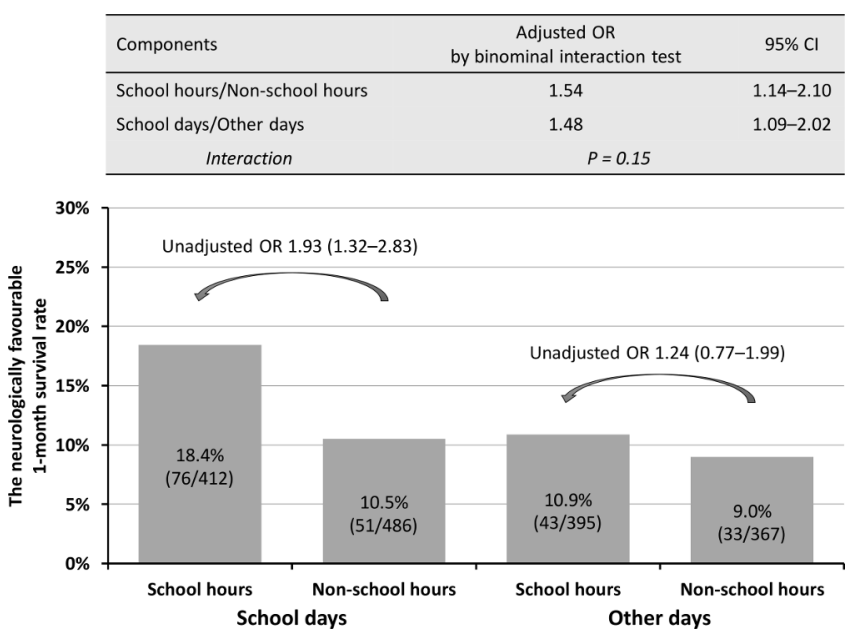

Figure 1 Interaction between school hours and school days and neurologically favourable 1-month survival.

of Ringer's lactate. Authorised and specially trained paramedics are permitted to insert tracheal tubes and administer intravenous epinephrine to patients with OHCA aged $\geq 8$ years under online medical direction by physicians. These paramedics are not allowed to administer drugs other than epinephrine. All EMS personnel resuscitate patients with OHCA in accordance with local protocols based on the Japan Resuscitation Council guidelines. $^{15}$

\section{Selection of participants}

The All-Japan Utstein Registry of FDMA includes Utstein-style information. ${ }^{16}$ The timelines of patient collapse and bystander CPR (BCPR) initiation were assessed by EMS personnel interviews with bystanders. The cardiac or non-cardiac cause of cardiac arrest was clinically evaluated by physicians in collaboration with EMS personnel. Fire departments obtained information on 1-month survival from hospitals. The FDMA logically evaluated data in the registry system, and if necessary, requested the respective fire department to correct and complete the data.

This study analysed bystander-witnessed patients with OHCA in school-age children groups without any physician involvement. As stated in the Utstein Recommendations, ${ }^{16}$ this implies that the non-physician bystander was present when the patient went into cardiac arrest before EMS arrival to the patient. We extracted the data for 1660 cases of bystander-witnessed OHCA in schoolchildren transported to hospitals by EMS that were managed without a physician and had a complete data set for analysis.

\section{Outcome measure}

The primary endpoint was the neurologically favourable 1-month survival, defined as cerebral performance category 1 or $2 .^{17}$

\section{Analysis}

School hours were defined as 08:00 to 18:00. Japan is composed of 47 prefectures, and school days in each prefecture were confirmed by excluding weekends, national and school holidays, and vacations. We assessed the incidence of OHCA and the neurologically favourable 1-month survival during school hours or other times of day (non-school hours) on both school days and non-school days (other days). To evaluate the interaction between school hours and school days, we applied a binominal interaction test. We then analysed differences in the survival rates between subgroups using univariate analyses, followed by propensity-matching procedures. Multivariable logistic regression analysis was also performed in a stepwise manner using the Akaike information criterion to identify major factors associated with neurologically favourable 1-month survival. The following factors were included in both propensity score matching and stepwise logistic regression: patient sex and age (classification of students), family or non-family bystanders, aetiology of OHCA (presumed cardiac or non-cardiac), initial ECG rhythm (shockable or non-shockable), BCPR (provided or not), call-first action by a bystander (time interval between emergency call and BCPR $<1 \mathrm{~min}$ ), public access defibrillation (PAD), tracheal intubation and epinephrine administration by paramedics, time interval between witness and first CPR performed by bystander or EMS personnel, whichever was earlier (witness-to-first CPR interval) and the time interval between emergency call and EMS arrival (EMS response time). Exogenous causes of OHCA were defined as trauma, submersion, poisoning and suicide attempts.

Differences between groups for nominal variables were assessed using the $\chi^{2}$ test and those for continuous variables were assessed using the Wilcoxon rank-sum test. The Cochrane-Armitage trend test was used to evaluate significant variations during the study period.

We analysed all data using JMP Pro V.12 (SAS Institute, Cary, NC). In each analysis, the null hypothesis was evaluated at a two-sided significant level of $\mathrm{p}<0.05$, with $95 \%$ CIs calculated using the profile likelihood.

\section{RESULTS}

\section{Impact of school hours and school days on OHCA survival}

Based on the analysis of 1660 cases of bystander-witnessed OHCA in schoolchildren, school hours and school days were associated with a neurologically favourable 1-month survival: adjusted OR (95\% CI) by binominal interaction test, 1.54 (95\% CI 1.14 to 2.10 ) and 1.48 (95\% CI 1.09 to 2.02 ), respectively. However, no significant interaction was observed between the two components $(p=0.15)$ (the upper part of figure 1$)$. The neurologically favourable 1-month survival rate during school hours was higher than that during non-school hours only on school days: $18.4 \%$ vs $10.5 \%$ on school days (unadjusted OR 1.93 ; $95 \%$ CI 1.32 to 2.83 ), $10.9 \%$ vs $9.0 \%$ on other days (unadjusted OR 1.24; 95\% CI 0.77 to 1.99 ) (figure 1). Furthermore, there were no significant differences among non-school hours on school days, school hours on other days and non-school hours on other days. Accordingly, we performed further analyses after dividing the groups into school hours on school days and all other times of day (non-school hours on school days and all times on other days).

Trends in AED installation at schools, and PAD proportion and OHCA outcomes during school hours on school days

The trends in cumulative proportion of AED installation at schools, biennial proportion of first defibrillations by bystanders in OHCA cases with prehospital defibrillation and OHCA outcomes are shown in figure 2. AEDs have been installed at almost all schools, and the PAD (first prehospital defibrillations by bystanders) proportion during school hours on school days significantly increased during the study period: $p<0.01$ by the Cochrane-Armitage trend test. However, the neurologically favourable 1-month survival rate during school hours on school days was not significantly improved: $p=0.21$. Although the neurologically favourable 1-month survival rate represented by the line in figure 2C slightly increased after the 2009-2010 
A

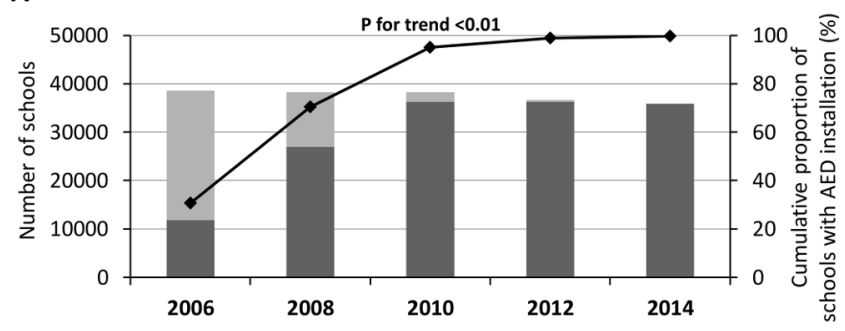

AED installation No AED installation $\rightarrow$ Proportion of schools

B

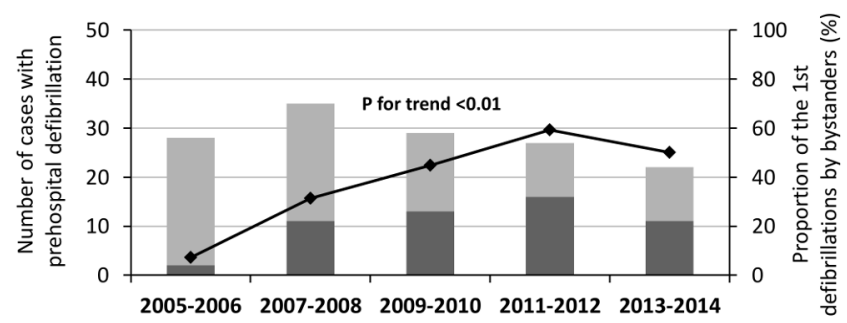

1st defibrillations by bystanders 1st defibrillations by EMS $\multimap$ PAD proportion

C

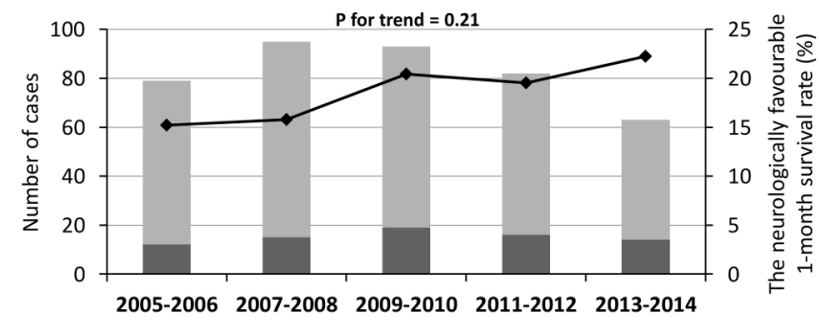

Neurologically favourable 1-month survival $=$ Others $\rightarrow$-The rate of survival

Figure 2 Trends in AED installation at schools, PAD proportion and OHCA outcomes during school hours on school days. (A) Left axis for stacked bar chart represents the total number of schools at the end of each year: the dark portion of the bar reflects the number of schools with AED installation. Right axis for line graph represents the cumulative proportion of schools with AED installation at the end of each year. (B) Left axis for stacked bar chart represents the total number of OHCA cases with prehospital defibrillation during the 2 years period: the dark portion of the bar reflects the number of cases with first defibrillations by bystanders (equally PAD). Right axis for line graph represents the proportion of the first defibrillations by bystanders during the 2 years period. The proportion of the first defibrillations by bystanders was defined as [number of OHCA cases with first defibrillation by bystanders] / [total number of OHCA cases with prehospital defibrillation]. (C) Left axis for stacked bar chart represents the total number of OHCA during the 2 years period. Right axis for line graph represents the neurologically favourable 1-month survival rate of OHCA during the 2 years period. AED, automated external defibrillator; EMS, emergency medical service; OHCA, out-of-hospital cardiac arrest; PAD, public access defibrillation.

period, the stepwise logistic regression analyses showed no significant increase: adjusted OR 1.76 ; 95\% CI 0.95 to 3.33 .

\section{Differences in OHCA outcomes between school hours on school days and all other times of day}

Before propensity score matching, a shockable initial rhythm and presumed cardiac aetiology, and shorter witness-to-first CPR interval and EMS response time were noted for OHCA cases during school hours on school days, and they more frequently received
BCPR, especially conventional BCPR, and PAD. However, callfirst action by a bystander was less frequent in these cases (table 1). The neurologically favourable 1-month survival rate during school hours on school days was significantly higher than those during all other times of day (table 2).

After propensity score matching, there were no significant differences in background, characteristics, time factors or survival outcomes between school hours on school days and all other times of day according to univariate analyses (tables 1 and 2).

\section{Factors associated with neurologically favourable 1-month survival}

Stepwise logistic regression analysis in OHCA cases during school hours on school days after propensity score matching revealed that a shockable initial rhythm (adjusted OR 2.44; 95\% CI 1.12 to 5.42), PAD (adjusted OR 3.32; 95\% CI 1.23 to 9.10), non-exogenous causes (adjusted OR 5.88; 95\% CI 1.85 to 20.0) and a shorter EMS response time (adjusted OR 1.15; 95\% CI 1.02 to 1.32 ) and witness-to-first CPR interval (adjusted OR 1.08; 95\% CI 1.01 to 1.15) were major factors associated with an improved neurologically favourable 1-month survival rate (figure 3). During all other times of day, a shockable initial rhythm (adjusted OR 4.81; 95\% CI 2.13 to 11.45 ) and PAD (adjusted OR 9.91; 95\% CI 3.86 to 27.60 ) were major factors associated with an improved neurologically favourable 1-month survival rate.

\section{DISCUSSION}

The survival outcomes of OHCA in schoolchildren were analysed based on school days and school hours. The neurologically favourable 1-month survival rate during school hours was better than that during non-school hours only on school days. When further analyses were performed after dividing the groups into school hours on school days and all other times of day, there was a significant difference in neurologically favourable survival between school hours on school days and all other times of day. However, the neurologically favourable survival did not significantly differ by logistic regression analysis after propensity score matching including backgrounds and characteristics of OHCA, demonstrating that school hours and school days are not independent factors associated with neurologically favourable survival from OHCA in schoolchildren.

According to the classification of students, the incidence of OHCA during school hours on school days was high among higher grades of elementary school and junior high school, whereas the incidence of OHCA among lower grades of elementary school and high school was higher during all other times of day than school hours on school days. This difference may have been due to the increased proportion of playing and participating in sports outside the school among lower grade elementary and high school students. Consistent with previous studies, ${ }^{346}$ the cause of OHCA during school hours on school days was more frequently presumed to be cardiac aetiology and the initial ECG rhythm recorded by AED was more frequently shockable than those during all other times of day. Furthermore, the proportion of PAD and BCPR was higher during school hours on school days. A significant difference was noted in the proportion of BCPR, but the difference was not large: during school hours on school days, $57.3 \%$; all other times of day, 43.6\%; unadjusted OR 1.74 ; $95 \%$ CI 1.39 to 2.17 . CPR combined with ventilations was more frequently performed than chest compression-only CPR, compared with all other times of day, which is a noteworthy difference. These resuscitation efforts by bystanders may contribute, at least in part, to the improved 
Table 1 Differences in background, characteristics and time factors for out-of-hospital cardiac arrest before and after propensity score matching for neurologically favourable 1-month survival

\begin{tabular}{|c|c|c|c|c|c|c|}
\hline \multirow[b]{2}{*}{$\begin{array}{l}\text { Background, characteristics and time } \\
\text { factors }\end{array}$} & \multicolumn{2}{|c|}{ Before propensity score matching } & \multirow{2}{*}{$\begin{array}{l}\text { Unadjusted OR } \\
(95 \% \mathrm{Cl}) \text { with all } \\
\text { other times of day as } \\
\text { reference or } \mathrm{P} \text { value }\end{array}$} & \multicolumn{2}{|c|}{ After propensity score matching } & \multirow{2}{*}{$\begin{array}{l}\text { Unadjusted OR } \\
(95 \% \mathrm{Cl}) \text { with all } \\
\text { other times of day as } \\
\text { reference or } \mathrm{P} \text { value }\end{array}$} \\
\hline & $\begin{array}{l}\text { School hours on } \\
\text { school days } \\
(n=412)\end{array}$ & $\begin{array}{l}\text { All other times } \\
\text { of day } \\
(n=1248)\end{array}$ & & $\begin{array}{l}\text { School hours on } \\
\text { school days } \\
(n=366)\end{array}$ & $\begin{array}{l}\text { All other times } \\
\text { of day } \\
(n=366)\end{array}$ & \\
\hline \multicolumn{7}{|l|}{ Classification of students, \% (n) } \\
\hline Lower grades in elementary school & $8.0(33)$ & $13.9(173)$ & $<0.01$ & $9.0(33)$ & $9.3(34)$ & 0.95 \\
\hline High school & $43.0(177)$ & $47.1(588)$ & & $44.5(163)$ & $42.4(155)$ & \\
\hline Family bystander-witnessed OHCA, \% (n) & $22.1(91)$ & $43.0(536)$ & $0.38(0.29$ to 0.49$)$ & $24.9(91)$ & $25.7(94)$ & 0.96 (0.69 to 1.34$)$ \\
\hline Presumed cardiac aetiology, \% (n) & $42.0(173)$ & $28.6(357)$ & 1.81 (1.43 to 2.28$)$ & $37.2(136)$ & $35.8(131)$ & 1.06 (0.79 to 1.43$)$ \\
\hline Exogenous causes, \% (n) & $39.1(161)$ & $52.6(656)$ & 0.58 (0.46 to 0.73$)$ & $43.4(159)$ & $44.0(161)$ & 0.98 (0.73 to 1.31$)$ \\
\hline Shockable initial rhythm, \% (n) & $23.5(97)$ & $15.8(197)$ & 1.64 (1.25 to 2.16$)$ & $21.6(79)$ & $21.6(79)$ & 1.00 (0.70 to 1.42$)$ \\
\hline DA-CPR provision, \% (n) & $34.5(142)$ & $34.3(428)$ & 1.01 (0.80 to 1.27 ) & $34.4(126)$ & $33.6(123)$ & 1.04 (0.76 to 1.41$)$ \\
\hline Call-first action by a bystander, $\%$ ( $\mathrm{n}$ ) & $59.7(141 / 236)$ & $71.1(387 / 544)$ & 0.61 (0.44 to 0.84$)$ & $58.4(115 / 197)$ & $65.7(132 / 201)$ & $0.76(0.50$ to 1.15$)$ \\
\hline Public access defibrillation, \% (n) & $12.9(53)$ & $3.3(41)$ & 4.35 (2.84 to 6.64$)$ & $7.4(27)$ & $10.1(37)$ & $0.71(0.42$ to 1.19$)$ \\
\hline Tracheal intubation by paramedics, \% (n) & $2.7(11)$ & $3.3(41)$ & 0.81 (0.41 to 1.59$)$ & $2.7(10)$ & $3.3(12)$ & 0.83 (0.35 to 1.94$)$ \\
\hline $\begin{array}{l}\text { Epinephrine administration by paramedics, } \\
\%(n)\end{array}$ & $7.3(30)$ & $4.4(54)$ & 1.72 (1.09 to 2.74$)$ & $6.3(23)$ & $5.7(21)$ & 1.10 (0.60 to 2.03$)$ \\
\hline \multicolumn{7}{|l|}{ Time intervals (min), median (IQR) } \\
\hline Witness-to-first CPR interval & $4(1-10)$ & $8(2-13)$ & $<0.01$ & $5(1-11)$ & $6(1-11)$ & 0.20 \\
\hline EMS response time & $7(6-9)$ & $8(6-10)$ & $<0.01$ & $7(6-9)$ & $7(6-10)$ & 0.10 \\
\hline
\end{tabular}

${ }^{*} \mathrm{CPR}$ with chest compressions and mouth-to-mouth ventilations.

BCPR, bystander CPR; CC, chest compression;CPR, cardiopulmonary resuscitation; DA-CPR, dispatcher-assisted CPR; EMS, emergency medical service; OHCA, out-of-hospital cardiac arrest.

survival outcome during school hours on school days, as reported for all OHCA cases. ${ }^{18}$

In Japan, the installation of AEDs in public facilities and buildings has rapidly increased since their use by citizens was approved in July 2004. The rate of bystander-witnessed OHCA with ventricular fibrillation and PAD was $1.1 \%$ in 2005, but it increased to $16.5 \%$ in $2013 .{ }^{19}$ In this study, as shown in figure $2 \mathrm{~A}$, the cumulative proportion of schools with AED installation was approximately $30 \%$ at the end of 2006 , but it has increased to nearly $100 \%$ at the end of 2012 . The biennial proportion of PAD in OHCA cases with prehospital defibrillation was $7 \%$ between 2005 and 2006, but it increased to approximately 50\% between 2013 and 2014 for OHCA in schoolchildren, as shown in figure 2B. However, the neurologically favourable 1-month survival rate did not improve despite the increased AED installation and PAD proportion (figure 2C). Consequently, the other factors were suggested to be related to poor survival outcome during school hours on school days.
In addition to PAD, two time intervals, witness-to-first CPR interval and EMS response time were identified as factors associated with neurologically favourable 1-month survival during school hours on school days in this study. These two time intervals were major factors associated with neurologically favourable outcome of all OHCA cases in schoolchildren, as reported in our preliminary analysis. ${ }^{20}$ This study revealed that a 1 min delay in CPR and EMS arrival during school hours on school days reduces the neurologically favourable 1 -month survival rate by $13 \%$ and $7 \%$, respectively. In contrast, these time factors did not influence the survival outcomes during all other times of day. Therefore, resolution of the causes influencing the time factors observed only during school hours on school days may be important to further improve the survival outcomes. BLS training for school staff and students is useful, ${ }^{21-23}$ but the main aim tended to be set at the quality of chest compressions and the use of the AED. Several causes may be considered for the delay in the witnessto-first CPR interval. Call-first action by bystanders decreased

Table 2 Out-of-hospital cardiac arrest outcomes before and after propensity score matching

\begin{tabular}{|c|c|c|c|c|c|c|}
\hline \multirow[b]{2}{*}{ Outcomes } & \multicolumn{2}{|c|}{ Before propensity score matching } & \multirow{2}{*}{$\begin{array}{l}\text { Unadjusted OR } \\
(95 \% \mathrm{CI}) \text { with all } \\
\text { other times of day as } \\
\text { reference }\end{array}$} & \multicolumn{2}{|c|}{ After propensity score matching } & \multirow{2}{*}{$\begin{array}{l}\text { Unadjusted OR } \\
(95 \% \mathrm{CI}) \text { with all } \\
\text { other times of day as } \\
\text { reference }\end{array}$} \\
\hline & $\begin{array}{l}\text { School hours on } \\
\text { school days } \\
\text { ( } n=412)\end{array}$ & $\begin{array}{l}\text { All other times of } \\
\text { day } \\
(n=1248)\end{array}$ & & $\begin{array}{l}\text { School hours on } \\
\text { school days } \\
(n=366)\end{array}$ & $\begin{array}{l}\text { All other times } \\
\text { of day } \\
(n=366)\end{array}$ & \\
\hline $\begin{array}{l}\text { Neurologically favourable } \\
\text { 1-month survival, } \% \text { ( })\end{array}$ & $18.4(76)$ & $10.2(127)$ & 2.00 (1.47 to 2.72 ) & $16.4(60)$ & $16.1(59)$ & 1.02 (0.69 to 1.51$)$ \\
\hline
\end{tabular}


School hours on school days

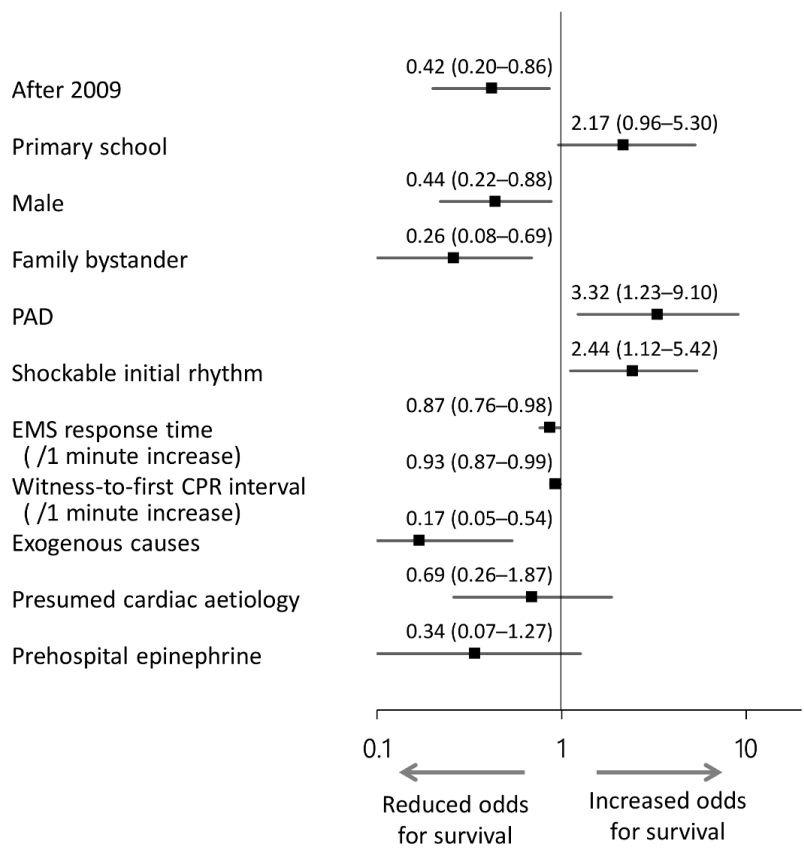

All other times of day

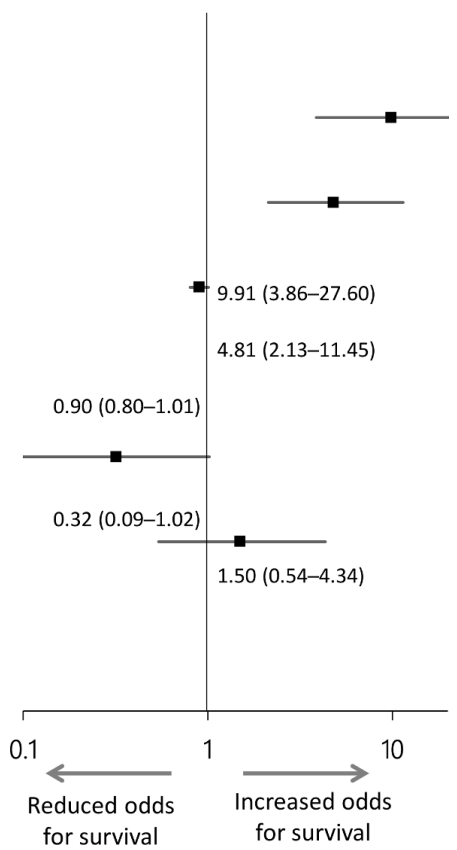

Figure 3 Factors associated with neurologically favourable 1-month survival. To identify major factors associated with neurologically favourable 1-month survival, we performed multivariable logistic regression analyses in a stepwise manner using the Akaike information criterion. CPR, cardiopulmonary resuscitation; EMS, emergency medical service; PAD, public access defibrillation.

during school hours on school days, suggesting that this was the cause of delay in BLS for patients with OHCA. Agonal breathing or gasping during collapse is likely to occur in cardiac causes with an initial ECG rhythm of ventricular fibrillation and lower age children, ${ }^{82}$ and school staff and students may misrecognise it as non-cardiac arrest, delaying the initiation of CPR. ${ }^{25}$ Emergency calls made using mobile phones were reported to affect the survival from OHCA by minimising the delay in emergency call and improving dispatcher-assisted CPR instruction. ${ }^{26}$ However, the use of a mobile phone by students at school is not permitted at many schools in Japan, and when the witness is a student, they go for other students and school staff, which delays the emergency response system and CPR may not be immediately initiated. Bystanders may also prioritise AED attachment before the emergency call and initiation of chest compressions, termed 'AED first'. ${ }^{27}$ If BCPR is not performed, CPR will not be initiated until the arrival of EMS personnel, and may be significantly delayed depending on the EMS response time.

The tolerance to hypoxaemia is poor in children compared with that in adults because the cerebral blood flow and metabolic needs are higher. ${ }^{28}$ The initial shockable rhythm at which PAD is effective and a shorter duration of CPR have been reported to be important factors involved in paediatric survival with a good outcome (neurological or hospital discharge). ${ }^{58}$ On the other hand, the benefits of early hypothermia for children in postresuscitation care have not been established, unlike for adults. ${ }^{528}$ Therefore, early and highquality BLS in a prehospital setting may be more important than in-hospital treatment for paediatric OHCA compared with adult OHCA in order to prevent cardiac arrest-associated neuronal loss, which may influence neurologically favourable outcomes.

The quality of BCPR and bystander-initiated resuscitation efforts for BLS are affected by the number of rescuers involved in providing BLS. ${ }^{29}$ There may be fewer rescuers during times other than school hours on school days at school, likely reducing the survival outcome. For BLS education at school, in addition to the ability to voluntarily perform high-quality $\mathrm{CPR}$ as the only rescuer, taking measures to increase the proportion of BCPR by training ${ }^{30}$ and learning to recognise cardiac arrest early are important. When no BCPR or high-quality BCPR or PAD is performed for bystander-witnessed OHCA in schoolchildren, the EMS response time may strongly influence the survival outcome. Although OHCA in schoolchildren accounts for only a small ratio of all cases of OHCA, increasing AED installation, and improving educational approaches for school staff members to facilitate early initiation of CPR and defibrillation by bystanders and EMS may improve the neurologically favourable survival outcome in schoolchildren.

Our study has several limitations. First, the statistical analysis was limited because of the small number of OHCA cases. Second, the association of the place of OHCA occurrence, such as at school, or during exercise or sports activity, was not investigated. Third, witnesses of OHCA at schools were not separated into school staff and students. As information regarding location was not available, it is possible that not all OHCA cases

\section{Key messages}

What is already known about this subject?

- Early initiation of cardiopulmonary resuscitation (CPR) is an important determinant of survival after out-of-hospital cardiac arrest in school-age children.

What does this study add?

- The time delays in CPR and emergency medical service (EMS) arrival during school hours on school days are independently associated with poorer outcomes.

How might this impact on clinical practice?

- Universal access to call EMS is important, in addition to the early recognition of cardiac arrest to initiate bystander CPR. 
during school hours on school days occurred at school. OHCA witnessed by families was included in this study. Thus, it is not possible to apply the study results to epidemiological evaluation of OHCA in schoolchildren occurring at school.

\section{CONCLUSIONS}

School hours are not an independent factor associated with improved outcomes of OHCA in schoolchildren. The time delays in CPR and EMS arrival were independently associated with poorer neurologically favourable 1-month outcome during school hours on school days. Early bystander and EMS-performed BLS based on sufficient preparedness may improve the outcomes of OHCA in schoolchildren.

Acknowledgements We thank the EMS personnel and Fire and Disaster Management Agency in Japan for their cooperation in collecting and managing the All-Japan Utstein Registry.

Funding The authors have not declared a specific grant for this research from any funding agency in the public, commercial or not-for-profit sectors.

Competing interests None declared.

Patient consent for publication Not required.

Ethics approval Ishikawa Medical Control Council Review Board

Provenance and peer review Not commissioned; externally peer reviewed.

\section{REFERENCES}

1 Lotfi K, White L, Rea T, et al. Cardiac arrest in schools. Circulation 2007;116:1374-9.

2 Atkins DL, Everson-Stewart S, Sears GK, et al. Epidemiology and outcomes from out-of-hospital cardiac arrest in children: the resuscitation outcomes Consortium Epistry-Cardiac arrest. Circulation 2009;119:1484-91.

3 Bardai A, Berdowski J, van der Werf C, et al. Incidence, causes, and outcomes of outof-hospital cardiac arrest in children. A comprehensive, prospective, population-based study in the Netherlands. J Am Coll Cardiol 2011:57:1822-8.

4 Nitta M, Iwami T, Kitamura T, et al. Age-Specific differences in outcomes after out-ofhospital cardiac arrests. Pediatrics 2011;128:e812-20.

5 Atkins DL, Berger S. Improving outcomes from out-of-hospital cardiac arrest in young children and adolescents. Pediatr Cardiol 2012;33:474-83.

6 Nishiuchi T, Hayashino Y, Iwami T, et al. Epidemiological characteristics of sudden cardiac arrest in schools. Resuscitation 2014;85:1001-6.

7 Hazinski MF, Markenson D, Neish S, et al. Response to cardiac arrest and selected life-threatening medical emergencies: the medical emergency response plan for schools: a statement for healthcare providers, policymakers, school administrators, and community leaders. Circulation 2004;109:278-91.

8 Berg MD, Schexnayder SM, Chameides L, et al. Part 13: pediatric basic life support: 2010 American heart association guidelines for cardiopulmonary resuscitation and emergency cardiovascular care. Circulation 2010;122(18 Suppl 3):S862-\$875.

9 Smith CM, Colquhoun MC. Out-Of-Hospital cardiac arrest in schools: a systematic review. Resuscitation 2015;96:296-302.

10 Taniguchi T, Omi W, Inaba H. Attitudes toward automated external defibrillator use in Japan. Resuscitation 2008;79:288-91.
11 Drezner JA, Rao AL, Heistand J, et al. Effectiveness of emergency response planning for sudden cardiac arrest in United States high schools with automated external defibrillators. Circulation 2009;120:518-25.

12 Cave DM, Aufderheide TP, Beeson J, et al. Importance and implementation of training in cardiopulmonary resuscitation and automated external defibrillation in schools: a science Advisory from the American heart association. Circulation 2011;123:691-706.

13 Swor R, Grace H, McGovern H, et al. Cardiac arrests in schools: assessing use of automated external defibrillators (AED) on school campuses. Resuscitation 2013:84:426-9.

14 Fire and disaster management agency (Japan), 1999. Available: http://www.fdma.go. jp/html/data/tuchi1107/110706kyu_176.htm; [Accessed 14 Sept 2001].

15 Japan Resuscitation Council. Available: http://qqzaidan.jp/pdf_5/guideline2_ALS_eng. pdf [Accessed 14 Sep 2001].

16 Jacobs I, Nadkarni V, Bahr J, et al. Cardiac arrest and cardiopulmonary resuscitation outcome reports: update and simplification of the Utstein templates for resuscitation registries: a statement for healthcare professionals from a task force of the International liaison Committee on resuscitation. Circulation 2004;110:3385-97.

17 Safar P. Resuscitation after brain ischemia. In: Grenvik A, Safar P, eds. Brain failure and resuscitation. New York: Churchill Livingstone, 1981: 155-80.

18 Yamashita A, Maeda T, Myojo Y, et al. Temporal variations in dispatcher-assisted and bystander-initiated resuscitation efforts. Am J Emerg Med 2018;36:2203-10.

19 Kitamura T, Kiyohara K, Sakai T, et al. Public-Access defibrillation and out-of-hospital cardiac arrest in Japan. N Engl J Med Overseas Ed 2016;375:1649-59.

20 Inaba H. Are school hours associated with better outcomes of out-of-hospital cardiac arrest in school children? A propensity-matched cohort study. Resuscitation 2018;130:e24

21 Kanstad BK, Nilsen SA, Fredriksen K. Cpr knowledge and attitude to performing bystander CPR among secondary school students in Norway. Resuscitation 2011;82:1053-9.

22 Bohn A, Van Aken HK, Möllhoff T, et al. Teaching resuscitation in schools: annual tuition by trained teachers is effective starting at age 10. A four-year prospective cohort study. Resuscitation 2012;83:619-25.

23 Stroobants J, Monsieurs K, Devriendt B, et al. Schoolchildren as BLS instructors for relatives and friends: impact on attitude towards bystander CPR. Resuscitation 2014;85:1769-74.

24 Clark JJ, Larsen MP, Culley LL, et al. Incidence of agonal respirations in sudden cardiac arrest. Ann Emerg Med 1992;21:1464-7.

25 Bång A, Herlitz J, Martinell S. Interaction between emergency medical dispatcher and caller in suspected out-of-hospital cardiac arrest calls with focus on agonal breathing. A review of 100 tape recordings of true cardiac arrest cases. Resuscitation 2003;56:25-34.

26 Maeda T, Yamashita A, Myojo Y, et al. Augmented survival of out-of-hospital cardiac arrest victims with the use of mobile phones for emergency communication under the DA-CPR protocol getting information from callers beside the victim. Resuscitation 2016:107:80-7.

27 Nishi T, Takei Y, Kamikura T, et al. Improper bystander-performed basic life support in cardiac arrests managed with public automated external defibrillators. Am J Emerg Med 2015;33:43-9.

28 Tress EE, Kochanek PM, Saladino RA, et al. Cardiac arrest in children. J Emerg Trauma Shock 2010;3:267-72.

29 Takei Y, Nishi T, Matsubara H, et al. Factors associated with quality of bystander CPR: the presence of multiple rescuers and bystander-initiated CPR without instruction. Resuscitation 2014;85:492-8.

30 Plant N, Taylor K. How best to teach CPR to schoolchildren: a systematic review. Resuscitation 2013;84:415-21. 